\title{
Step effects on diffusion near a substrate reconstructive phase transition: $H$ on $W(100)$
}

\author{
Lei Cai, ${ }^{1}$ Chaozhi Zheng, ${ }^{1}$ K. L. Man, ${ }^{1}$ M. S. Altman, ${ }^{1}$ E. Granato, ${ }^{2}$ T. Ala-Nissila,${ }^{3,4}$ S. C. Ying, ${ }^{3}$ and Xudong Xiao ${ }^{1, *}$ \\ ${ }^{1}$ Department of Physics, The Hong Kong University of Science and Technology, Hong Kong, SAR, China \\ ${ }^{2}$ Instituto Nacional de Pesquisas Espaciais, Sao Jose dos Campos, Sao Paolo, Brazil \\ ${ }^{3}$ Department of Physics, Brown University, Providence, Rhode Island 02912 USA \\ ${ }^{4}$ Helsinki Institute of Physics and Laboratory of Physics, Helsinki University of Technology, P.O. Box 1100, \\ FIN-02015 HUT, Espoo, Finland
}

(Received 13 December 2002; published 29 August 2003)

\begin{abstract}
We have used the linear optical diffraction method to study the diffusion of hydrogen atoms on flat and stepped W(100) surfaces. At 0.17-monolayer (ML) H coverage, the diffusion coefficient $(D)$ shows a strong anomalous dip at the substrate reconstructive phase transition temperature in an Arrhenius plot for diffusion on both surfaces. No anomalous diffusion behavior is observed at 1.2-ML H coverage on both surfaces in the entire range studied, 240-380 K, consistent with the absence of the phase transition at this $\mathrm{H}$ coverage. The strong reduction of $D$ can be attributed to the diverging friction damping near the transition. Steps do not suppress the substrate phase transition and affect the diffusion anomaly very little. For both $\mathrm{H}$ coverages, the only effect of steps is to introduce a small Schwoebel-Ehrlich barrier $(\sim 2.2 \mathrm{kcal} / \mathrm{mol}$ for $1.2 \mathrm{ML}$ and $\sim 2.8$ $\mathrm{kcal} / \mathrm{mol}$ for $0.17 \mathrm{ML}$ ) near the step edges, which slows down $\mathrm{H}$ diffusion perpendicular to steps. Measurements of $\mathrm{H}$ diffusion parallel to steps reveals no obvious enhancement due to step edge diffusion.
\end{abstract}

DOI: 10.1103/PhysRevB.68.075422

PACS number(s): 68.43.Jk, 68.35.Rh, 68.35.Fx, 68.35.Ja

\section{INTRODUCTION}

Surface diffusion plays an important role in many interesting surface processes, such as adsorption, catalysis, and film growth. Changes in the structure of a substrate may have a considerable effect on surface diffusion. For example, surface diffusion near an adsorbate or substrate phase transition is expected to have anomalous non-Arrhenius temperature dependence based on theoretical grounds. ${ }^{1-3}$ Experimentally, such behavior has often been observed near adsorbate layer phase transitions and is sometimes even used to identify surface phase transitions. ${ }^{4-6}$ However, the underlying physics for such observations is rarely understood, mostly due to the lack of knowledge of the nature of the relevant phase transitions. In contrast, the nature of the substrate reconstructive phase transition of the $\mathrm{W}(100)$ surface is well understood. ${ }^{7-16}$ It provides an ideal model system to investigate the effect of the substrate phase transition on surface diffusion. As reported in a recent Letter, ${ }^{17}$ the $\mathrm{H}$ diffusion anomaly near the reversible phase transition of $\mathrm{W}(100)$ from a $(1 \times 1)$ disordered phase at high temperature to a $c(2$ $\times 2$ ) phase at low temperature was clearly demonstrated by experiment, in qualitative agreement with the prediction of an earlier theoretical work. ${ }^{1}$

Surface steps, even at low densities, can have a considerable effect on the surface diffusion process. ${ }^{18}$ This is another example showing that changes in substrate (an increase in step density) can have significant effects on surface diffusion. In a recent study of $\mathrm{CO}$ diffusion on vicinal $\mathrm{Pt}(111)$ surfaces, ${ }^{19-21} \mathrm{Ma}$ and co-workers observed new and very interesting diffusion behavior related to steps. For diffusion perpendicular to steps, the diffusion was impeded by an extra step trapping potential well rather than an extra step barrier (Schwoebel-Ehrlich barrier). ${ }^{20}$ For diffusion parallel to steps, in addition to the channels over the terrace and along the step edges, a new channel was observed that enhanced diffusion by about one order of magnitude. ${ }^{21}$ Step effects, which are unavoidable in any macroscopic measurement that probes an area containing a large number of steps, can sometimes mask the true surface diffusion process such as intrinsic terrace diffusion. In this paper, we therefore extend our earlier study of the $\mathrm{H}$ diffusion anomaly near the $\mathrm{W}(100)$ substrate phase transition ${ }^{17}$ to the stepped surface, in part to investigate possible step effects on the anomalous behavior of $D$ near the phase transition.

The $\mathrm{W}(100)(1 \times 1)$ surface has a fourfold symmetry and the diffusion of $\mathrm{H}$ on it is therefore expected to be isotropic. However, the reconstructed $c(2 \times 2)$ structure has a twofold symmetry and diffusion of $\mathrm{H}$ on it is expected to be anisotropic. For the clean surface and the surface with very low $\mathrm{H}$ coverage [less than 0.12 monolayer (ML)], the structure of the reconstruction at low temperature was originally deduced by symmetry arguments to consist of atomic displacements along the diagonal $\langle 110\rangle$ surface directions to form zigzag chains. ${ }^{9,14}$ The displacement magnitude of the top-layer atoms on the clean surface has been determined to be 0.22 $\AA^{15,22}$ The low-energy electron diffraction (LEED) pattern of the $c(2 \times 2)$ structure shows fourfold symmetry due to contributions from two orthogonal degenerate domains whose symmetries are only twofold $(p 2 m g)$. When the hydrogen coverage exceeds about $0.12 \mathrm{ML}$, a change of the $c(2 \times 2)$ structure symmetry from $p 2 m g$ of the clean surface to $c 2 \mathrm{~mm}$ is induced. ${ }^{10,13,14,23}$ From this change, it was deduced that hydrogen causes $\mathrm{W}$ atom displacements to switch from along the $\langle 110\rangle$ surface directions to along the $\langle 100\rangle$ surface directions ${ }^{10,14}$ to form a dimerlike structure. Again, there are two types of domains that are related by a rotation of $90^{\circ}$ about the surface normal. They are equivalent and coexist with about equal probability on a flat $\mathrm{W}(100)$ surface. The degeneracy of the two types of domains possibly renders the diffusion isotropic when averaged over a macroscopic measurement length scale of micrometers, which is much 
larger than the microscopic domain size. However, the degeneracy of the two types of domains can be lifted by properly orienting and spacing surface steps. ${ }^{10,14,23-26}$ For example, a domain ratio of about 30:1 was reported for the $p 2 m g$ structure on the clean surface as a result of miscutting the surface along the [110] direction by $3.25^{\circ}$, which resulted in a terrace width about $28 \AA^{23,27}$ The atomic displacements in the $p 2 \mathrm{mg}$ reconstructed structure are preferentially perpendicular to the step edges for this miscut. ${ }^{23,25}$ For the $c 2 \mathrm{~mm}$ structure, miscutting a surface along the [100] direction, which results in steps along the surface [010] direction, can break the degeneracy and results in preferential domains with displacements of the $\mathrm{W}$ atoms along the step edge. ${ }^{10,13,14,26} \mathrm{~A}$ second purpose of our experiment will be to study the $\mathrm{H}$ diffusion anisotropy on single-type domains that are obtained on a stepped $\mathrm{W}(100)$ surface.

The organization of the paper is as follows. We will first describe how the experiment is performed. Then, the results of $\mathrm{H}$ diffusion on a stepped $\mathrm{W}(100)$ surface together with those for a flat surface will be presented. The results on the flat surface have been published in a recent Letter ${ }^{17}$ and are included here for comparison. Diffusion both perpendicular and parallel to steps will be covered. In the Discussion section, we will discuss the step effect on the diffusion anomaly near the $\mathrm{W}(100)$ reconstruction phase transition, followed by a discussion of the diffusion across and along steps. The anisotropy effect of $\mathrm{H}$ diffusion on a single-domain phase will also be briefly discussed. The experimental results show that the step effects on both the diffusion anomaly and diffusion anisotropy are small. This nontrivial observation by itself is already interesting and will stimulate further theoretical study.

\section{EXPERIMENTAL DETAILS}

The experiments were carried out in a stainless steel UHV system with a base pressure of $\sim 2 \times 10^{-10}$ torr. Two singlecrystal W(100) samples were used: one was cut and mechanically polished to within $0.1^{\circ}$ of the (100) plane; the other was cut $2.5^{\circ}$ off the (100) plane in the [010] direction. A $95 \% \mathrm{~W}-5 \% \mathrm{Re} / 74 \% \mathrm{~W}-26 \%$ Re thermocouple was spotwelded to the side of the crystals to measure the sample temperature. Each sample was first cleaned by cycles of annealing in oxygen at $1480 \mathrm{~K}$ and $2 \times 10^{-7}$ torr for $20 \mathrm{~min}$, and subsequent flashing to $2000 \mathrm{~K}$ until Auger spectra showed no detectable surface impurities. The total time of oxygen treatment was more than $5 \mathrm{~h}$. Observations of the $c(2 \times 2)$ LEED patterns of the reconstructed surface at low temperatures also indicated that the sample was clean and well ordered. On the stepped surface, the integer LEED spots were elongated, with the amount of elongation quantitatively confirming the step density introduced by the miscut. After the sample was cleaned of bulk contaminants, the surface was routinely cleaned by high-temperature (2000-K) flashing only prior to each individual diffusion run. The sample could be cooled by liquid nitrogen (LN) from 2000 to $90 \mathrm{~K}$ in about 3 min. Adsorption of $\mathrm{H}$ on the $\mathrm{W}(100)$ surface was carried out at approximately $90 \mathrm{~K}$ by leaking $\mathrm{H}_{2}$ gas into the chamber. The $\mathrm{H}$ coverage was calibrated by thermal desorp- tion spectroscopy (TDS) on the flat surface. Because the W atoms are known to be immobile at temperatures below 200 $\mathrm{K},{ }^{10}$ the sample was slowly heated to room temperature and held for about 2 min to equilibrate the surface. It was known from other studies that hydrogen adsorption is dissociative and that hydrogen atoms adsorb on the bridge sites between $\mathrm{W}$ atoms at all coverages. ${ }^{28-33}$ The saturation coverage of $\mathrm{H}$ on $\mathrm{W}(100)$ is two monolayers $\left(2 \times 10^{15}\right.$ adatoms/ $\left.\mathrm{cm}^{2}\right){ }^{13,34-37}$

The diffusion coefficient $D$ was measured using a linear optical diffraction technique, ${ }^{38}$ which has several advantages: (a) a wide dynamic range for measuring the diffusion coefficient, (b) a high adsorbate coverage sensitivity, and (c) an intrinsic capability for measuring diffusion anisotropy. In this method, first, an adsorbate grating is created by laser-induced thermal desorption with two interfering pulsed laser beams. In our experiment, we used a grating period of 3.9 or $6.7 \mu \mathrm{m}$ to measure the diffusion coefficient $D$ slower or faster than $\sim 10^{-9} \mathrm{~cm}^{2} / \mathrm{s}$, respectively, and a shallow coverage modulation of about $0.03 \mathrm{ML}$ to ensure the diffusion coefficient $D$ in the relevant coverage range to be well-approximated as a constant. Once such a grating is produced, the first-order diffraction of a He-Ne laser beam with polarization modulation is used to probe the smearing of the grating induced by surface diffusion. The diffraction signal decays exponentially according to ${ }^{38}$

$$
S=S(0) \exp (-t / \tau), \quad \tau=s^{2} / 8 \pi^{2} D .
$$

Here, $D$ is the chemical diffusion coefficient and $s$ is the grating period. To determine $D$, only the decay time constant and the grating period were needed. The detailed shape of the grating would not affect $D$. In our study, diffusion measurements were carried out between 200 and $450 \mathrm{~K}$ at two different $\mathrm{H}$ coverages (1.2 and 0.17 ML). On the stepped surface, diffusion both perpendicular and parallel to the steps was measured with the adsorbate grating appropriately oriented.

\section{RESULTS}

The diffusion coefficients at 1.2-ML $\mathrm{H}$ coverage on flat and stepped surfaces are depicted in the Arrhenius plot in Fig. 1. On the flat surface, the diffusion coefficient can be fitted by the simple Arrhenius law $D=D_{0} \exp \left(-E_{\text {diff }} / k_{B} T\right)$ over the entire temperature range, with a prefactor $D_{0}$ $\sim 10^{-(2.2 \pm 0.2)} \mathrm{cm}^{2} / \mathrm{s}$ and a diffusion activation energy $E_{\text {diff }}$ $\sim 10.5 \pm 0.3 \mathrm{kcal} / \mathrm{mol}$. On the highly stepped surface, it is seen that the steps, even at a high density of 1 step/12 terrace rows, do not affect the $\mathrm{H}$ diffusion to a large extent. Within the experimental error, diffusion parallel to steps is essentially the same as that on the flat surface. Diffusion perpendicular to steps is slightly slower than that on the flat surface, perhaps reflecting a somewhat larger diffusion barrier across the steps, as will be discussed later.

The diffusion data at 0.17-ML H coverage on the flat and stepped $\mathrm{W}(100)$ surfaces are shown in Fig. 2. $D_{0}$ $\sim 10^{-(1.2 \pm 0.4)} \mathrm{cm}^{2} / \mathrm{s}$ and $E_{\text {diff }} \sim 11.7 \pm 0.5 \mathrm{kcal} / \mathrm{mol}$ are obtained from fitting the diffusion data on the flat surface below $325 \mathrm{~K}$. On both the flat and stepped surfaces, the diffusion 


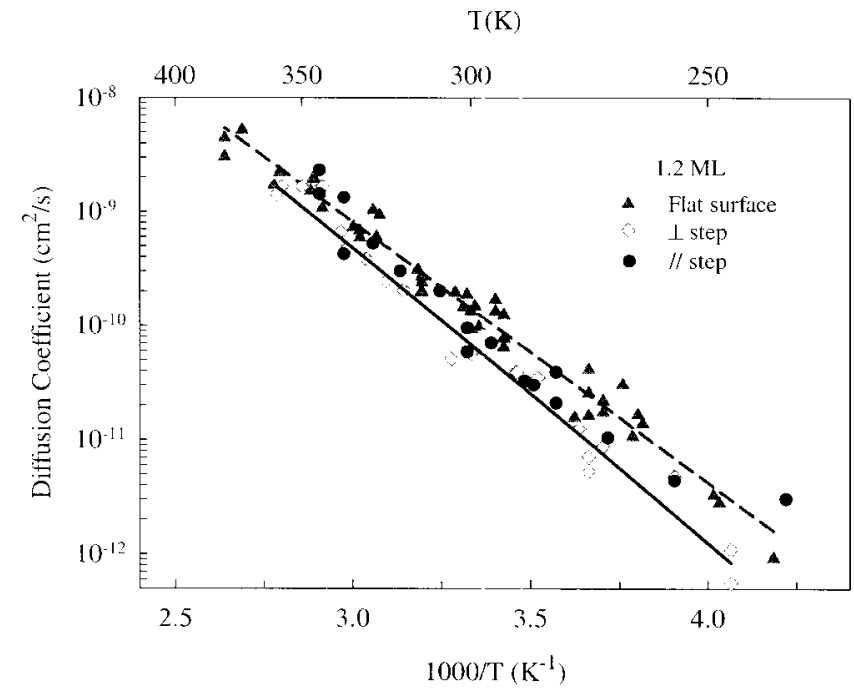

FIG. 1. H diffusion coefficient $D$ on the W(100) surface vs reciprocal temperature $1 / T$ at $1.2-\mathrm{ML} \mathrm{H}$ coverage on flat and stepped surfaces. The flat sample is polished to within $0.1^{\circ}$ from the (100) plane, and the stepped sample is miscut $2.5^{\circ}$ of the (100) plane in the [010] direction. The short-dashed line is a fitting by the simple Arrhenius law $D=D_{0} \exp \left(-E_{\text {diff }} / k_{B} T\right)$. The solid line is a fitting by Eq. (2) for diffusion perpendicular to steps.

coefficient appears to have a pronounced dip around $355 \mathrm{~K}$. The dip on the flat surface was found to correlate with $T_{C}$ determined from LEED measurements. Within experimental error, the behavior of the dip is identical on the flat and stepped surfaces. While the diffusion coefficient parallel to steps is basically the same as that on the flat surface, the diffusion coefficient perpendicular to steps deviates somewhat from that on the flat surface and is most likely related to the Schwoebel-Ehrlich barrier effect.

For completeness, the diffusion data at 0.08 - and $0.31-\mathrm{ML}$

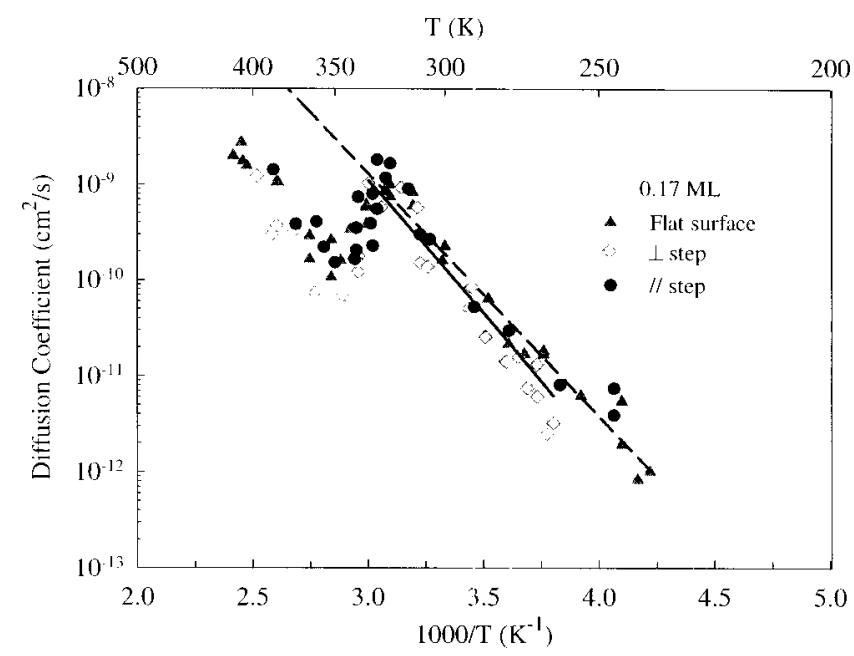

FIG. 2. $D$ vs $1 / T$ at $0.17-\mathrm{ML} H$ coverage on flat and stepped surfaces. For the latter diffusion both perpendicular and parallel to steps was measured. Below the temperature of $325 \mathrm{~K}$, the diffusion data on the flat surface can be fitted by $D=D_{0} \exp \left(-E_{\text {diff }} / k_{B} T\right)$ (dashed line), and the diffusion data perpendicular to steps can be fitted by Eq. (2) (solid line).

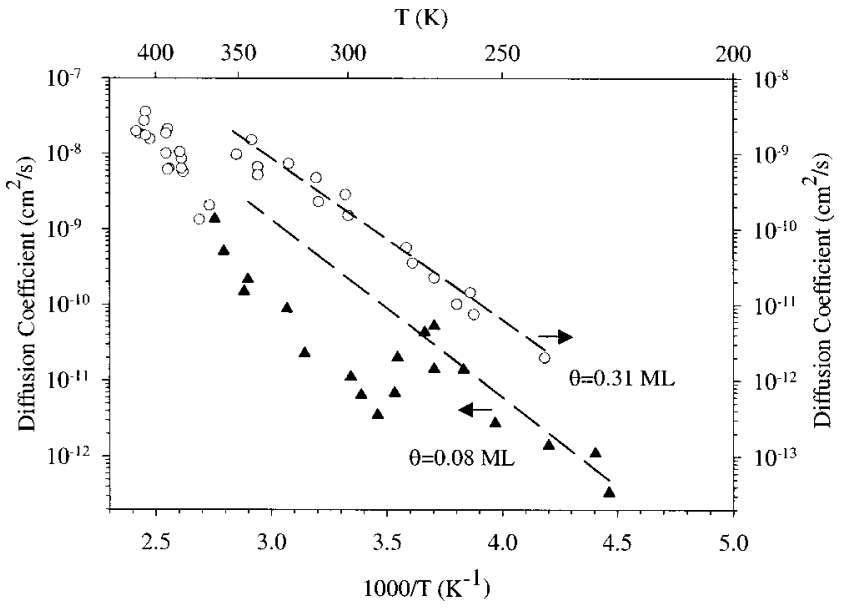

FIG. 3. $D$ vs $1 / T$ at 0.08 - and $0.31-\mathrm{ML} \mathrm{H}$ coverage on flat surface. The dashed lines are fittings by $D=D_{0} \exp \left(-E_{\text {diff }} / k_{B} T\right)$ at temperatures below the transition.

$\mathrm{H}$ coverages on the flat $\mathrm{W}(100)$ surface (the former were reported in our previous Letter ${ }^{17}$ ), are included in Fig. 3. The behavior of $D$ is similar to that for $0.17 \mathrm{ML}$, except that the dip of $D$ occurs at a temperature near $290 \mathrm{~K}$ at $0.08 \mathrm{ML}$ and near $365 \mathrm{~K}$ at $0.31 \mathrm{ML}$, corresponding to the different $T_{C}$ at these coverages. The diffusion data in the low-temperature range can be fitted by $D_{0} \sim 10^{-(1.9 \pm 1.2)} \mathrm{cm}^{2} / \mathrm{s}$ and $E_{\text {diff }}$ $\sim 10.8 \pm 1.4 \mathrm{kcal} / \mathrm{mol}$ at $0.08 \mathrm{ML}$ and $D_{0} \sim 10^{-(2.8 \pm 0.6)}$ $\mathrm{cm}^{2} / \mathrm{s}$ and $E_{\text {diff }} \sim 9.9 \pm 0.5 \mathrm{kcal} / \mathrm{mol}$ at $0.31 \mathrm{ML}$.

\section{DISCUSSION}

\section{A. Phase transition effect}

While the step density on the flat surface could be higher than that determined by the miscut $\left(<0.1^{\circ}\right)$ due to a possible hill-and-valley morphology developed through repetitive high temperature annealing, the size of the LEED spots on the flat surface remains limited by instrument resolution and the corresponding step density cannot be deduced. On the stepped surface, however, the integer LEED spots are clearly elongated, with the amount of elongation consistent with a terrace size of $\sim 36 \AA$. The size of the LEED spot in the orthogonal direction is the same as that on the flat surface, with an instrument-limited dimension at least three times smaller than that in the elongated direction. Thus, the step density on the stepped surface must at least be three times higher than that on the flat surface, limited by the ability of measuring the true step density on the latter. This independent evidence on different step densities for the flat and stepped surfaces should validate our following discussion on the step effects.

As shown in Fig. 1, no anomalous behavior of $D$ is observed both on the flat and stepped surfaces at 1.2-ML $\mathrm{H}$ coverage. This is consistent with the fact that there is no substrate phase transition over the entire temperature range at this coverage. ${ }^{39}$ At $\mathrm{H}$ coverages of $0.08,0.17$, and $0.31 \mathrm{ML}$, however, an anomalous decrease of $D$ with increasing temperature is obvious as shown in Figs. 2 and 3. Our LEED 
measurements ${ }^{17}$ and the previous study of the H/W(100) phase diagram ${ }^{10}$ indicate that the dip of the diffusion coefficient occurs at a temperature that is consistent with the coverage-dependent substrate phase transition temperature $T_{C}$.

The origin of the anomalous diffusion behavior near the substrate phase transition temperature has been discussed in our previous Letter. ${ }^{17}$ Near the reconstructive phase transition of $\mathrm{W}(100)$, the vibrational modes near the critical wave vector $\mathbf{q}_{0}=(\pi / a, \pi / a)$ soften and become overdamped as $T$ approaches $T_{C}$. The anomalous temperature dependence of the substrate phonons leads to critical singularities in the dynamic structure factor $S(\mathbf{q}, \omega)$ near $T_{C}$ and ultimately to an anomaly in the temperature dependence of the friction acting on the $\mathrm{H}$ adatoms. Near $T_{C}, S(\mathbf{q}, \omega)$ obeys the scaling form $\xi_{c}^{z+\gamma / \nu} g_{ \pm}\left(\left|\mathbf{q}-\mathbf{q}_{0}\right| \xi_{c}, \omega \xi_{c}^{z}\right)$, where $g_{ \pm}$is a scaling function, $\xi_{c} \propto\left|T / T_{C}-1\right|^{-\nu}$ is the divergent correlation length, $\gamma$ is the susceptibility exponent, and $z$ is the dynamical critical exponent. ${ }^{3}$ In the Markovian limit of instantaneous damping, the frictional damping $\eta$ is simply determined by the average of $S(\mathbf{q}, \omega=0)$ over a range of $\mathbf{q}$ determined by the coupling potential of the $\mathrm{H}$ adatom to the substrate. ${ }^{1,3}$ This leads then to a $\eta$ diverging as $\left|T-T_{C}\right|^{-x}$ with $x=\nu(z-d)+\gamma$ (in $d$ dimensions) and $D \sim \eta^{-1}$ vanishing as $T$ approaches $T_{C}$.

The simple argument presented above only applies when $T$ is not too close to $T_{C}$. Two additional factors need to be considered, which eliminate the infinite divergence of the frictional damping and result in a finite dip in $D$ instead of it vanishing at $T_{C}$. The first is the breakdown of the Markovian approximation that the frictional damping is proportional to the instantaneous velocity. ${ }^{40,41}$ As one approaches $T_{C}$, the dynamic structure factor $S(\mathbf{q}, \omega)$ gets narrower and stronger and develops into a "central peak" structure. ${ }^{3}$ When the width of this central peak gets narrower than the characteristic frequency for the motion of the $\mathrm{H}$ adatom, the time scale of the relevant substrate excitations is longer than the time scale of the motion of the hydrogen adatom. At this point, the instantaneous damping picture breaks down and the divergence in the frictional damping is cut off. The second factor that suppresses divergence is sample imperfection such as finite size or a finite step density. Since the phases of the reconstruction for the domains on each terrace are uncorrelated, divergence of the correlation length $\xi$ is cut off when it reaches the average size of a reconstructed domain. ${ }^{42}$ Thus the finite frequency of the $\mathrm{H}$ adatom acts as a temporal cutoff, while the finite size of the reconstructed domain acts as a spatial cutoff that can be described by appropriate finitesize scaling theory. ${ }^{3,41}$ The rounding of the anomaly and the elimination of the true divergence of the friction are due to whichever effect that first becomes dominant as one approaches $T_{C}$. For the "flat" surface, the terrace size is at least $100 \AA$ and it is most likely that the non-Markovian effect, i.e., the temporal cutoff, is responsible for the rounding of the anomaly. On the stepped surface, the average size of the reconstructed domains is even smaller than the average terrace width since experimentally, there is evidence that the $\mathrm{W}(100)$ reconstruction may be inhibited near step edges at low temperatures. ${ }^{13,24,25,27,43,44}$ The degree of the inhibition is described by the inhibition range. The most reliable measurements of this quantity obtained from LEED spot profile analysis ${ }^{24,25,27}$ indicate that both the clean and $\mathrm{H}$-induced structures are inhibited over a range that does not exceed $8 \AA$ in the proximity of steps along the surface [110] or [010] directions. The $2.5^{\circ}$ miscut of our sample implies a terrace width of only about $36 \AA$. Thus the size of the reconstructed domain is of order of $28 \AA$. The observation of $\left(\frac{1}{2} \frac{1}{2}\right)$ LEED spots and the dip in the plot of $D$ vs $1 / T$ at 0.17 ML (Fig. 2) indicates that the surface of the $2.5^{\circ}$ miscut W(100) substrate still undergoes a reconstructive phase transition. Since there is little difference in the dip of $D$ between that on the flat surface and that on the stepped surface, this implies that on the stepped surface, either the temporal cutoff is still responsible for the rounding of the anomaly, or else the temporal and spatial cutoff occur at a similar temperature region around $T_{C}$. At this point, we do not have enough information on the detailed parameters of the system to distinguish between these two scenarios.

\section{B. Schwoebel-Ehrlich barrier and effective coverage}

It is well known that steps may affect diffusion perpendicular to steps through a Schwoebel-Ehrlich barrier ${ }^{45-47}$ and/or an additional trapping potential well at the step edges. ${ }^{18,20}$ Diffusion parallel to steps may be affected by a fast channel along the steps. ${ }^{18,20}$ Both theoretical and experimental studies have shown these effects.

Diffusion perpendicular to steps must be subject to several processes in sequence. They are diffusion over a terrace, over a step edge barrier (i.e., Schwoebel-Ehrlich barrier) to reach a trapping site, and hopping out of the trapping site to the adjacent terrace site. The diffusion time for these sequential processes is additive. Based on the lattice gas model with nearest-neighbor hopping, Merikoski and Ying have analyzed these processes in detail. ${ }^{18}$ For a Langmuir gas with no adsorbate-adsorbate interaction except site blocking, the overall diffusion coefficient perpendicular to steps $D_{\perp}$ in the limit of no additional trapping barrier $\left(\Delta E_{B} \sim 0\right)$ at the step sites is given by ${ }^{18}$

$\frac{1}{D_{\perp}}=\frac{L-2}{L} \frac{1}{\nu_{t} a^{2} e^{-E_{t} / k_{B} T}}+\frac{1}{L} \frac{1}{\nu_{\mathrm{eff}} a^{2} e^{-\left(E_{t}+\Delta E_{s}\right) / k_{B} T}}$,

where

$$
\nu_{\mathrm{eff}}=\frac{\nu_{s}}{1+\left(\nu_{s} / \nu_{B}\right) e^{-\Delta E_{s} / k_{B} T}},
$$

$L$ is the terrace width in units of the lattice constant $a, \nu_{t}$ and $E_{t}$ are the attempt frequency and activation energy on a terrace, respectively, $\nu_{S}$ and $\nu_{B}$ are the attempt frequencies over the Schwoebel-Ehrlich barrier and out of the trapping well, respectively, and $\Delta E_{S}$ is the height of the Schwoebel-Ehrlich barrier. Thus, $D_{t}=\nu_{t} a^{2} \exp \left(-E_{t} / k_{B} T\right)$ is the terrace diffusion coefficient and $D_{s}=\nu_{\text {eff }} a^{2} \exp \left[-\left(E_{t}+\Delta E_{S}\right) / k_{B} T\right]$ is the diffusion coefficient over steps.

We fit our data for 1.2- and 0.17-ML $\mathrm{H}$ coverage on the stepped surface with Eq. (2) assuming the values of $D_{0, t}$ $=\nu_{t} a^{2}$ and $E_{t}$ are the same as those on the flat surface. Here, we limit the data range for $0.17 \mathrm{ML}$ only in the low- 
TABLE I. The fitted values of the prefactor and diffusion activation energy at 1.2- and 0.17-ML $\mathrm{H}$ coverages.

\begin{tabular}{lcccc}
\hline \hline $\begin{array}{l}\text { Coverage } \\
(\mathrm{ML})\end{array}$ & $\begin{array}{c}D_{0, t} \\
\left(\mathrm{~cm}^{2} / \mathrm{s}\right)\end{array}$ & $\begin{array}{c}E_{t} \\
(\mathrm{kcal} / \mathrm{mol})\end{array}$ & $\begin{array}{c}D_{0, s} \\
\left(\mathrm{~cm}^{2} / \mathrm{s}\right)\end{array}$ & $\begin{array}{c}\Delta E_{S} \\
(\mathrm{kcal} / \mathrm{mol})\end{array}$ \\
\hline 1.2 & $10^{-(2.2 \pm 0.2)}$ & $10.5 \pm 0.3$ & $10^{-(1.8 \pm 0.6)}$ & $2.2 \pm 0.8$ \\
0.17 & $10^{-(1.2 \pm 0.4)}$ & $11.7 \pm 0.5$ & $10^{-(0.1 \pm 2.4)}$ & $2.8 \pm 3.2$ \\
\hline \hline
\end{tabular}

temperature region $(<325 \mathrm{~K})$. The fitting curves are shown in Figs. 1 and 2 as solid lines. The fitting values of $D_{0, s}$ $=\nu_{\text {eff }} a^{2}$ and $\Delta E_{S}$ are listed in Table I. We find $\Delta E_{S}$ changes a little for different $\mathrm{H}$ coverages. The values determined here for $\mathrm{H} / \mathrm{W}(100)$ are similar to the value $\Delta E_{S}=2.7 \mathrm{kcal} / \mathrm{mol}$ for $\mathrm{H}$ diffusion on a step $\mathrm{Ru}(001)$ surface determined by laserinduced thermal desorption techniques (LITD) and by assuming a negligible $\Delta E_{B} \cdot{ }^{48}$

For diffusion parallel to steps, the diffusion activation energy $E_{2}$ along the step edge may be different from that on terrace, which results in a different diffusion rate along the steps. In the previous theoretical studies of Merikoski and Ying, ${ }^{18}$ it was shown that the diffusion coefficient parallel to the step for the condition $\Delta E_{B} \sim 0$ is given by ${ }^{18}$

$$
D_{\|}=\frac{L-1}{L} D_{t}+\frac{1}{L} D_{s, \|},
$$

where $D_{s, \|}=D_{0,2} \exp \left(-E_{2} / k_{B} T\right)$ is the diffusion coefficient along the step edge. In both Figs. 1 and 2, we observe that the diffusion coefficient parallel to steps is basically the same as that on the terrace within the experimental error in the entire temperature range for 1.2- and 0.17-ML $\mathrm{H}$ coverages. According to Eq. (3), this means that the diffusion coefficient $\left(D_{s, \|}\right)$ along the step edge must be equal to or slower than the diffusion coefficient on the terrace $\left(D_{t}\right)$. Thus, steps do not introduce a fast channel to diffusion parallel to steps.

The present results are in strong contrast to $\mathrm{CO}$ diffusion on vicinal $\mathrm{Pt}(111)$ surfaces that were studied by our group previously. ${ }^{19-21}$ While diffusion of CO perpendicular to steps is impeded by a strong additional trapping potential at the step sites $\left(\Delta E_{B} \sim 7 \mathrm{kcal} / \mathrm{mol}\right)$, diffusion parallel to steps shows an enhancement over terrace diffusion in various ways. At high temperatures, all three types of steps, type $A$, $B$, and $A B$, show the contribution to mass transport by the fast step-edge diffusion. At low temperatures, a third channel, which exists only for $A$ - and $A B$-type steps, also contributes to diffusion enhancement. The enhancement of the diffusion coefficient due to steps is more than two orders of magnitude. In the present system of H/W(100), such step effects are absent or below the detection limit. The above comparison indicates that effects of the steps on the magnitude of surface diffusion depend strongly on the particular systems being studied. No universal rules about the step barriers across and along the steps can be deduced from the information available so far.

We must add a note of caution about the fits to the experimental data in Figs. 1 and 2. Aside from the magnitude of the Schwoebel-Erlich barrier and the fact that there are no fast channels parallel to the steps, the details of the fits cannot be taken too seriously because of the simplicity of the model. In particular, the existence of an inhibition range has not been taken into account explicitly. It has been shown by Lau and Ying $^{26}$ that on a reconstructed surface, the binding energy of the adsorption sites increases monotonically with the amplitude of the reconstruction. Thus, instead of additional trapping at the step edge, the hydrogen adatoms would actually stay away from the inhibition region and only adsorb and diffuse in the reconstructed domain. Depending on the inhibition range and hence the size of the reconstructed domain, the actual $\mathrm{H}$ coverage on the reconstructed domain can be significantly higher than the nominal coverage determined by the exposure over the entire surface. This could explain the fact that when we examined the LEED patterns at nominal 0.17-ML coverage on the stepped surface, we found that each $\left(\frac{1}{2} \frac{1}{2}\right)$ spot splits into two circular spots, perpendicular to the step directions. The splitting indicates the formation of an incommensurate structure that is known to occur on a flat surface at a coverage of about $0.3 \mathrm{ML}$, much higher than the nominal coverage of $0.17 \mathrm{ML}$ averaged over the whole surface.

\section{Anisotropy}

The adsorption of $\mathrm{H}$ on $\mathrm{W}(100)$ induces a substrate reconstruction phase transition from $(1 \times 1)$ at high temperatures to $c(2 \times 2)$ at low temperatures. The $c 2 \mathrm{~mm}$ symmetry of the $c(2 \times 2)$ structure at a $\mathrm{H}$ coverage between $\sim 0.12$ and $\sim 0.30 \mathrm{ML}$ has a twofold rotation symmetry in contrast to a fourfold rotation symmetry for the unreconstructed W(100) surface. Thus, diffusion on the $c(2 \times 2)$ surface may be expected to be anisotropic. On a flat reconstructed W(100) surface, the orthogonal degenerate domains will be present with equal probability. Therefore, the anisotropy of surface diffusion is averaged out in a macroscopic measurement that probes many domains. In contrast, the reconstruction is preferentially oriented with atomic displacements along steps on a stepped surface, ${ }^{13,14,23}$ which causes an unequal population of the two orthogonal domains. A strong domain preference was also observed here. From the $\left(\frac{1}{2} \frac{1}{2}\right)$ spot splitting (only two instead of four spots) on the stepped surface, it clearly shows single domain orientation. Thus, the diffusion anisotropy is expected to be observable on the stepped surface. This domain-induced diffusion anisotropy will be superimposed on the anisotropy due to steps. The diffusion anisotropy due to steps alone at 1.2-ML coverage is clearly shown in Fig. 1 and the amount of anisotropy is small. In Fig. 2, the fact that the diffusion results parallel to steps at $0.17-\mathrm{ML} \mathrm{H}$ coverage are nearly identical to the flat surface ones, which is a statistical average of diffusion over the two orthogonal domains with equal populations, indicates that the anticipated anisotropy is small. For diffusion perpendicular to steps, the diffusion data can be fitted well by Eq. (2) when we set $D_{0, t}$ and $E_{t}$ equal to the corresponding values on the flat surface, further supporting the notion that the diffusion anisotropy on the $c(2 \times 2)$ domain is small. However, this conclusion is subject to the uncertainty of the incommensurate structure which has a periodicity of $\sim 20 \AA$ along the steps determined from the splitting of the half order spots. How the domain wall affects diffusion anisotropy is not yet clear. 


\section{CONCLUSION}

We have investigated the step effect on $\mathrm{H}$ diffusion near the $\mathrm{W}(100)$ reconstructive phase transition. The anomalous dip of the diffusion coefficient $D$ observed in its Arrhenius plot, due to the diverging friction damping near the phase transition, is not significantly affected by the steps.

From this we conclude that the reconstruction phase transition still occurs on the surface with high step densities. The divergence of the length and time scale near $T_{C}$ are cut off due to either non-Markovian effects as in the case of the flat surface or the finite size of the reconstructed domain.

For diffusion parallel and perpendicular to the steps, dips of $D$ were observed similar to that on a flat W(100) surface. The step introduces a Schwoebel-Ehrlich barrier, about 2.2

*Author to whom correspondence should be addressed. Email address: phxudong@ust.hk

${ }^{1}$ T. Ala-Nissila, W. K. Han, and S. C. Ying, Phys. Rev. Lett. 68, 1866 (1992).

${ }^{2}$ I. Vattulainen, J. Merikoski, T. Ala-Nissila, and S. C. Ying, Phys. Rev. Lett. 79, 257 (1997).

${ }^{3}$ M. R. Baldan, E. Granato, and S. C. Ying, Phys. Rev. B 62, 2146 (2000).

${ }^{4}$ R. Gomer, Rep. Prog. Phys. 53, 917 (1990), and references therein.

${ }^{5}$ A. G. Naumovets, V. V. Poplavsky, and Yu. S. Vedula, Surf. Sci. 200, 321 (1988).

${ }^{6}$ S. C. Wong and R. Gomer, J. Chem. Phys. 83, 4193 (1985).

${ }^{7}$ K. Yonehare and L. D. Schmidt, Surf. Sci. 25, 238 (1971).

${ }^{8}$ T. E. Felter, R. A. Barker, and P. J. Estrup, Phys. Rev. Lett. 38, 1128 (1977).

${ }^{9}$ M. K. Debe and D. A. King, Phys. Rev. Lett. 39, 708 (1977).

${ }^{10}$ R. A. Barker and P. J. Estrup, J. Chem. Phys. 74, 1442 (1981).

${ }^{11}$ J. F. Wendelken and G. C. Wang, Phys. Rev. B 32, 7542 (1985).

${ }^{12}$ R. A. Barker, P. J. Estrup, F. Jona, and P. M. Marcus, Solid State Commun. 25, 375 (1978).

${ }^{13}$ D. A. King and G. Thomas, Surf. Sci. 92, 201 (1980).

${ }^{14}$ K. Griffiths, D. A. King, and G. Thomas, Vacuum 31, 671 (1981).

${ }^{15}$ C. L. Fu and A. J. Freeman, Phys. Rev. B 37, 2685 (1988).

${ }^{16}$ I. K. Robinson, A. A. MacDowell, M. S. Altman, P. J. Estrup, K. Evans-Lutterodt, J. D. Brock, and R. J. Birgeneau, Phys. Rev. Lett. 62, 1294 (1989).

${ }^{17}$ L. Cai, M. S. Altman, E. Granato, T. Ala-Nissila, S. C. Ying, X. Xiao, Phys. Rev. Lett. 88, 226105 (2002).

${ }^{18}$ J. Merikoski and S. C. Ying, Phys. Rev. B 56, 2166 (1997).

${ }^{19}$ J. Ma, X. Xiao, N. J. DiNardo, and M. M. T. Loy, Phys. Rev. B 58, 4977 (1998).

${ }^{20}$ J. Ma, L. Cai, X. Xiao, and M. M. T. Loy, Surf. Sci. 425, 131 (1999).

${ }^{21}$ J. Ma, X. Xiao, and M. M. T. Loy, Surf. Sci. 436, L661 (1999). $\mathrm{kcal} / \mathrm{mol}$ for $1.2-\mathrm{ML}$ and $2.8 \mathrm{kcal} / \mathrm{mol}$ for $0.17-\mathrm{ML} \mathrm{H}$ coverages, to influence $\mathrm{H}$ diffusion perpendicular to the steps. Diffusion parallel to steps has not been affected by the steps at all. From the measurements, we also conclude that the diffusion anisotropy on the $c(2 \times 2)$ domain is small.

\section{ACKNOWLEDGMENT}

We wish to acknowledge financial support from the Research Grant Council of Hong Kong through Grant No. HKUST6114/98P, the William Mong Solid State Cluster Laboratory (L.C., M.S.A. and X.X.), CNPq, and NSF grants (S.C.Y., E.G., and T.A.-N.), FAPESP (E.G.), and the Academy of Finland through its Center of Excellence program (T.A.-N.).
${ }^{22}$ M. S. Altman, P. J. Estrup, and I. K. Robinson, Phys. Rev. B 38, 5211 (1988).

${ }^{23}$ J. F. Wendelken and G.-C. Wang, J. Vac. Sci. Technol. A 3, 1593 (1985).

${ }^{24}$ J. K. Zuo, T. M. Lu, and G. C. Wang, J. Vac. Sci. Technol. A 5, 777 (1987).

${ }^{25}$ G.-C. Wang and T.-M. Lu, Surf. Sci. 122, L635 (1982).

${ }^{26}$ K. H. Lau and S. C. Ying, Phys. Rev. Lett. 44, 1222 (1980).

${ }^{27}$ J. F. Wendelken and G. C. Wang, Surf. Sci. 140, 425 (1984).

${ }^{28}$ E. A. Daniels and R. Gomer, Surf. Sci. 336, 245 (1995).

${ }^{29}$ A. Adnot and J. D. Carette, Phys. Rev. Lett. 39, 209 (1997).

${ }^{30}$ W. Ho, R. F. Willis, and E. W. Plummer, Phys. Rev. Lett. 40, 1463 (1978); 42, 12 (1978).

${ }^{31}$ R. F. Willis, Surf. Sci. 89, 457 (1979).

${ }^{32}$ M. R. Barnes and R. F. Willis, Phys. Rev. Lett. 41, 1727 (1978).

${ }^{33}$ A. H. Smith, R. A. Barker, and P. J. Estrup, Surf. Sci. 136, 327 (1984).

${ }^{34}$ P. J. Estrup and J. Andeerson, J. Chem. Phys. 45, 2254 (1966).

${ }^{35}$ T. E. Mandy, Surf. Sci. 36, 281 (1973).

${ }^{36}$ I. Stensgaard, L. C. Feldman, and P. Silverman, Phys. Rev. Lett. 42, 247 (1979).

${ }^{37}$ L. C. Feldman, P. Silverman, and I. Stensgaard, Surf. Sci. 87, 295 (1979).

${ }^{38}$ X. Xiao, Y. Xie, and Y. R. Shen, Surf. Sci. 271, 295 (1992).

${ }^{39}$ J. J. Arrecis, Y. J. Chabal, and S. B. Christman, Phys. Rev. B 33, 7906 (1986).

${ }^{40}$ A. Cucchetti and S. C. Ying, Phys. Rev. B 54, 3300 (1996).

${ }^{41}$ E. Falck, M.Sc. thesis, Helsinki University of Technology, 2001.

${ }^{42}$ M. E. Fisher and M. N. Barber, Phys. Rev. Lett. 28, 1516 (1972).

${ }^{43}$ T. M. Gardiner and E. Bauer, Surf. Sci. 119, L353 (1982).

${ }^{44}$ M. K. Debe and D. A. King, Surf. Sci. 81, 193 (1979).

${ }^{45}$ R. L. Schwoebel and E. J. Shipsey, J. Appl. Phys. 37, 3682 (1966).

${ }^{46}$ G. Ehrlich and F. G. Hudda, J. Chem. Phys. 44, 1039 (1966).

${ }^{47}$ R. L. Schwoebel, J. Appl. Phys. 40, 614 (1969).

${ }^{48}$ M. V. Arena, E. D. Westre, D. E. Brown, J. Kutzner, S. M. George, Surf. Sci. 325, 151 (1995). 